\title{
Evaluation de l'empreinte carbone d'une Installation de Stockage des Déchets Non Dangereux (ISDND) : Cas du centre traitement et de valorisation des déchets (CTVD) sis à Polesgo (Ouagadougou)
}

\section{Kayaba, HARO ${ }^{1,2, *}$; Issoufou, OUARMA ${ }^{2}$; Oumar, SANOGO ${ }^{1}$; COMPAORE, Abdoulaye $^{1}$; TUBREOUMYA, Guy Christian²; Bernard, NANA ${ }^{2,3}$; Antoine, BERE ${ }^{2}$ and Jean KOULIDIATI ${ }^{2}$}

\author{
${ }^{1}$ Institut de Recherche en Sciences Appliquées et Technologie (IRSAT), 01 BP : 7047 Ouagadougou 01 \\ ${ }^{2}$ Laboratoire de Physique et de Chimie de l'Environnement (LPCE), Université Joseph KI-ZERBO 03 BP : 7021 \\ Ouagadougou 03 \\ ${ }^{3}$ Institut Des Sciences (IDS) 01. BP : 1757 Ouagadougou 01 \\ kayabaharo@rocketmail.com
}

\section{INFOS SUR L'A R T I C L E}

Historique de l'article:

Reçu le : 31 juillet 2019

Reçu en format revisé le : 14 décembre 2019

Accepté le : 18 décembre 2019

\section{Mots-Clés:}

Dechet solides municipaux, gaz à effet de

serre, LandGEM

Keywords

Municipal solid waste, greenhouse gas, LandGEM

\section{R E S U M E / A B S T R A C T}

Résumé. Les Installations de Stockage des Déchets Non Dangereux (ISDND) produisent du biogaz par dégradation anaérobique de la fraction organique des déchets enfouis. Ce biogaz majoritairement composé de méthane $\left(\mathrm{CH}_{4}\right)$ et de dioxyde de carbone $\left(\mathrm{CO}_{2}\right)$, deux gaz à effet de serre, représente un enjeu environnemental majeur. Ainsi l'impact « effet de serre » engendré par une ISDND peut être évalué par son « empreinte carbone », laquelle est obtenue en quantifiant en équivalents dioxyde de carbone (éq$\mathrm{CO}_{2}$ ), les émissions de Gaz à Effet de Serre (GES), principalement le $\mathrm{CH}_{4}$ et le $\mathrm{CO}_{2}$. Cet article propose une estimation du bilan carbone du centre de traitement et de valorisation des déchets sis à Polesgo par modélisation. Cette estimation est réalisée en s'appuyant sur un modèle cinétique du premier ordre (LandGEM) qui tient compte de données in situ assurant une description suffisamment proche de la réalité.

\begin{abstract}
Landfill produce biogas by anaerobic degradation of the organic fraction of buried waste. This biogas mainly composed of methane $\left(\mathrm{CH}_{4}\right)$ and carbon dioxide $\left(\mathrm{CO}_{2}\right)$ represents a major environmental issue. Thus, greenhouse effect impact generated by a landfill can be evaluated by its carbon footprint, which is obtained by quantifying in carbon dioxide equivalents $\left(\mathrm{CO}_{2} \mathrm{e}\right)$, Greenhouse Gas $(\mathrm{GHG})$ emissions, mainly $\mathrm{CH}_{4}$ and $\mathrm{CO}_{2}$. This article provides an estimate of the carbon footprint of Polesgo's landfill by modeling. This estimate is based on a first-order kinetic model (LandGEM) that takes into account in situ data ensuring a sufficiently close description of reality.
\end{abstract}

\section{INTRODUCTION}

Le changement climatique est l'un des défis environnementaux majeur auquel l'humanité est confrontée. Il est à l'origine de l'élévation du niveau de la mer, de la fréquence accrue des cyclones et de la répartition inégale des précipitations, ce qui affecte la production agricole, la biodiversité et les cycles naturels. Ces perturbations sont attribuées au phénomène de réchauffement planétaire dû à l'émission globale des gaz à effet de serre (GES) tels que le $\mathrm{CO}_{2}$, le $\mathrm{CH}_{4}$ et le $\mathrm{N}_{2} \mathrm{O}$ provenant d'activités anthropiques. Plus de $60 \%$ des émissions totales de $\mathrm{CH}_{4}$ sont imputables aux activités humaines, dont les pratiques de gestion des déchets. La gestion des déchets solides représente de nos jours l'un des défis les plus
}

importants des sociétés urbaines et industrielles. Selon Guerrero et al., 2013 ces défis sont accentués par certains facteurs tels que la croissance démographique, l'urbanisation rapide et le développement socioéconomique des villes des pays en développement. Si dans les pays industrialisés l'enfouissement des déchets solides produits par les centres urbains et par les industries est une pratique courante et maîtrisée sur le plan technologique et s'ajoute à d'autres pratiques de valorisation matière et énergie, elle est la solution par défaut pratiquée sans aucune considération pour l'environnement dans les pays en développement. De grandes quantités de déchets sont encore enfouies de façon inadéquate, dans des décharges incontrôlées. Des efforts ont été fournis 
avec la création de centres d'enfouissement technique calqués sur les modèles internationaux mais ils fonctionnent très mal. Ces amas de déchets constituent encore des ressources potentielles de pollution aggravée $\mathrm{du}$ fait que les lixiviats produits sont généralement mal drainés et non traités et que le biogaz produit n'est ni récupéré, ni valorisé. A cela s'ajoutent les impacts visuels et olfactifs ainsi que les risques d'incendie. Cependant, ces centres d'enfouissement technique de déchets sont considérés comme des sources mineures de GES à l'échelle internationale et le plus souvent négligé à l'échelle nationale. Les raisons tiennent principalement au manque de données d'émission ou de génération de GES des centres d'enfouissement surtout dans les villes des pays du Sud comme le cas de Ouagadougou.

Quelques études ont été menées dans les pays développés afin de mettre en évidence la contribution effective des ISDND au phénomène de changement climatique. Ce qui permet de noter que la contribution globale des ISDND aux émissions des GES à l'atmosphère est estimée à 3-5\% des émissions globale des GES selon le rapport du programme des Nations Unies pour l'environnement (UNEP). C'est la deuxième source anthropogénique de méthane $\left(\mathrm{CH}_{4}\right)$ après l'agriculture (UNEP, 2010). Il contribue autour de $18 \%$ aux émissions de méthane dans l'air au niveau mondial (Bogner et al., 2008 ; Krautwurst et al., 2017 ; Pham et al., 2017 ; Gunnar et al., 2000) et 4,1\% au niveau national. Dans le contexte de lutte contre les changements climatiques, il parait plus que nécessaire d'identifier les potentielles sources de GES afin d'améliorer les stratégies d'atténuation des émissions des GES. C'est dans cette optique que s'inscrit cet article dont l'objectif principal est d'estimer la contribution du centre de traitement et de valorisation des déchets (CTVD) de Polesgo à l'effet de serre en lien avec l'enfouissement des déchets solides ménagers.

\section{MATERIELS ET METHODE}

\subsection{Présentation de la zone d'étude}

Le centre de traitement et de valorisation des déchets de Polesgo a été mis en service le 15avril 2005. Il a une capacité de 6,1 millions de mètre cubes $\left(\mathrm{m}^{3}\right)$ de déchets et offre une possibilité d'exploitation de vingt (20) ans (jusqu'en 2025). Il couvre une superficie de 70 hectares et est situé à une dizaine de kilomètres au nord de Ouagadougou dans l'arrondissement de NongrMaâssom. Le CTVD assure deux missions essentielles qui sont : (i) l'enfouissement des déchets solides ; (2i) la valorisation des déchets solides (compostage et valorisation du plastique). La figure 1 présente une vue aérienne du CTVD de Polesgo.

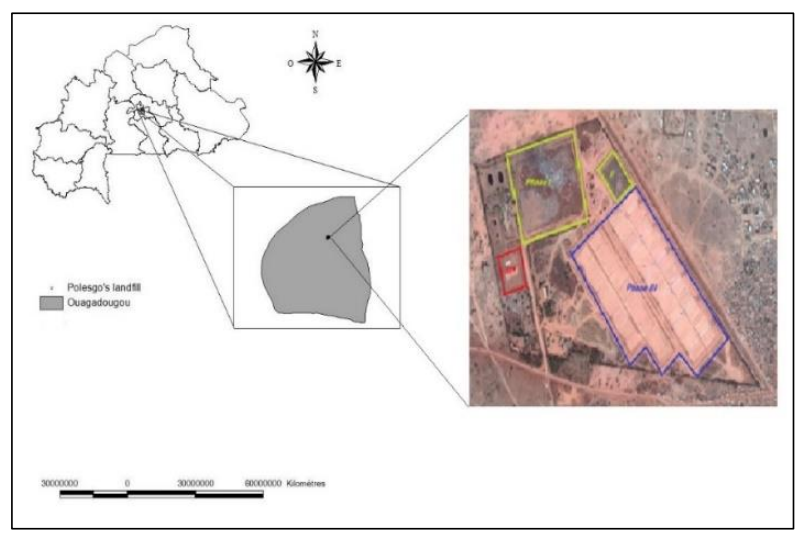

Fig.1 : Vue aérienne du CVTD de Polesgo

\subsection{Evaluation de la quantité de déchets enfouis au CTVD de Polesgo}

La quantité de Déchets Solides Municipaux (DSM) enfouis au CTVD de 2005 à 2018 a été fournie par la direction de la propreté de la commune de Ouagadougou. Celle de 2019 à 2025 a été extrapolée en utilisant les données démographiques de la ville de Ouagadougou, du taux de collecte des déchets solides municipaux et du taux d'enfouissement des déchets collectés. Le taux de croissance de la population et la production spécifique journalière de déchets sont respectivement de 7,6\% (INSD, 2017) et de 0,62 $\mathrm{kg} /$ habitant/jour (Tezanou et al., 2003). La population de Ouagadougou est d'environ 2,5 million d'habitants en 2018 selon la projection de l'Institut National de la Statistique et de la démographie (INSD). Selon une étude réalisée pour la Banque Mondiale, seulement $41 \%$ des déchets solides municipaux produits dans la ville de Ouagadougou sont collectés et acheminés au CTVD de Polesgo. Environ $90 \%$ de ces déchets acheminés sont enfouis dans les cellules d'enfouissement. Ainsi, la masse des déchets enfouis au CTVD $\left(\mathrm{M}_{\mathrm{F}}\right)$ est obtenue par l'équation (1):

$$
M_{F}=t_{c} \times t_{e} \times M_{T}
$$

Où :

$t_{c}$ est le taux de collecte des déchets $(0,41)$;

$t_{\mathrm{e}}$ est le taux d'enfouissement au CTVD de Polesgo

$M_{T}$ (t/an) est la masse totale de déchet généré par an. $M_{T}$ est calculée à partir de l'équation (2):

$$
M_{T}(t)=n_{j a} \times P(t) \times w_{c}
$$

$\mathrm{P}(\mathrm{t})$ la population attendue sur l'année considérée (de 2019 à 2025) en tenant compte d'un taux de croissance $\mathrm{r}$ de $4,1 \% ; w_{c}$ est la production spécifique journalière de déchets de $0,62 \mathrm{~kg} /$ habitant/jour ; $n_{j a}$ est le nombre de jours d'une année (365). Ainsi, la population attendue $P(t)$ est déterminée par l'équation (3) : 


$$
P(t)=P_{0} \times(1+r)^{t}
$$

Où :

$r$ : Taux de croissance

$P_{0}$ est la population de Ouagadougou de l'année 2018 prise comme référence ; t est le temps d'extrapolation (en année).La masse de déchets mise en décharge est calculée par l'équation (4):

$$
M_{F}=t_{c} \times t_{e} \times n_{j a} \times w_{c} \times P_{0} \times(1+r)^{t}
$$

\subsection{Evaluation de l'empreinte carbone du CTVD de Polesgo}

Le dioxyde de carbone et le méthane issus de la biodégradation des déchets sont les seuls gaz pris en compte dans cette étude. Des pouvoirs de réchauffement global (PRG) de 1 et 28 ont respectivement été considérés pour le $\mathrm{CO}_{2}$ et le $\mathrm{CH}_{4}$ (IPCC, 2013). Le $\mathrm{CO}_{2}$ et autre GES émanant des engins de transport des déchets et autres travaux sur le site n'ont pas été comptabilisés dans cette étude. L'empreinte carbone de CTVD a été évaluée à partir des deux composantes majoritaires $\left(\mathrm{CH}_{4}\right.$ et $\left.\mathrm{CO}_{2}\right) \mathrm{du}$ biogaz produit et est donnée par l'équation (5). Ces composants du biogaz sont évalués à l'aide du modèle cinétique du $1^{\mathrm{er}}$ ordre LandGEM V3.02 (Alexander $e t$ al., 2005).

$$
C_{1}=\varphi_{\mathrm{CH}_{4}} \times \rho_{\mathrm{CH}_{4}} \times P R G_{\mathrm{CH}_{4}}+\varphi_{\mathrm{CO}_{2}} \times \rho_{\mathrm{CO}_{2}} \times P R G_{\mathrm{CO}_{2}}
$$

Où :

$C_{1} \quad$ : Quantité de GES en tonne équivalent dioxyde de carbone (téqCO2);

$\varphi_{\mathrm{CH}_{4}} \quad: \quad$ Volume moyen en $\mathrm{m}^{3} / \mathrm{an}$ de méthane rejeté dans l'atmosphère ;

$\rho_{\mathrm{CH}_{4}} \quad: \quad$ Masse volumique en $\mathrm{kg} / \mathrm{m}^{3} \mathrm{du}$ méthane ;

$P R G_{C_{4}}$ : Pouvoir de réchauffement global du methane (=28 sur la base de 100 ans) ;

$\varphi_{\mathrm{CO}_{2}} \quad: \quad$ Volume moyen en $\mathrm{m}^{3}$ /an de dioxyde de carbone rejeté dans l'atmosphère ;

$\rho_{\mathrm{CO}_{2}}: \begin{aligned} & \text { Masse volumique en } \\ & \text { dioxyde de carbone } ;\end{aligned}$

$P R G_{\mathrm{CO}_{2}}$ : Pouvoir de réchauffement global du dioxyde de carbone $(=1)$.

\section{a) Présentation du modèle LandGEM}

Le modèle LandGEM a été développé par l'agence américaine pour la protection de l'environnement (USEPA) pour la prédiction de la génération des polluants gazeux dans les centres de stockage des déchets solides non dangereux. Il considère (i) les quantités et les caractéristiques des déchets enfouis sur plusieurs années consécutives, (ii) les caractéristiques du biogaz produit et (iii) les conditions météorologiques de la zone d'étude. Il permet la prédiction de la génération de $\mathrm{CH}_{4}$ et de $\mathrm{CO}_{2}$ jusqu'à 140 ans (après l'ouverture du CTVD) à partir d'une équation de décomposition du $1^{\mathrm{er}}$ ordre (Lagos, 2014). L'équation de génération de $\mathrm{CH}_{4}$ (Equation 6) considère des incréments du dixième (1/10) d'année dans le but d'augmenter le niveau de précision de l'estimation.

$$
Q_{C H_{4}}=\sum_{i=1}^{N} \sum_{j=0,1}^{1} k \times L_{0} \times\left(\frac{M_{i}}{10}\right) \times e^{-k t_{i, j}}
$$

Où

$Q_{\mathrm{CH}_{4}} \quad$ : $\quad$ Volume annuel en $\mathrm{m}^{3}$ de méthane produit ;

$N \quad$ : Nombre total d'années concernées par la

simulation numérique ;

$i \quad: \quad$ Indice relatif à la $i^{\text {ème }}$ année concernée par la

$i \quad$ simulation numérique ;

$k \quad: \quad$ Constante de dégradation des déchets (1/an);

$L_{0} \quad$ : $\quad$ Potentiel de production de méthane en $\mathrm{m}^{3}$ par unité de masse ;

M : $\quad$ Masse de déchets enfouis en $\mathrm{Mg}$ dans la $\mathrm{i}$ ème année ;

$\mathrm{t}_{\mathrm{i}, \mathrm{j}} \quad$ : $\quad$ Âge de la jième section de la fraction $i$ de déchets enfouis dans la i ème année.

\section{b) Paramètres du modèle LandGEM}

Il existe deux types de paramètres du modèle LandGEM : (i) les paramètres par défaut fournis par l'US EPA et (ii) les paramètres in situ déterminés à partir des caractéristiques des déchets enfouis et des conditions météorologiques du site d'étude (Alexander et al., 2005). Dans la présente étude, ce sont les paramètres in situ déterminés par Haro et al., 2017 (Tableau 1) qui ont été utilisés.

\section{Tableau3. Paramètres de LandGEM}

\begin{tabular}{|c|c|c|}
\hline Constante & Valeurs in situ & $\begin{array}{c}\text { Valeurs } \\
\text { par défaut }\end{array}$ \\
\hline Lo & $98,03^{[\mathrm{a}]}$ & $100^{[\mathrm{b}]}$ \\
\hline $\mathrm{k}$ & $0,035^{[\mathrm{a}]}$ & $0,02^{[\mathrm{b}]}$ \\
\hline
\end{tabular}

\section{RESULTATS ET DISCUSSIONS}

Les figures 2 et 3 montrent l'évolution et la contribution cumulée de l'empreinte carbone au fil des années du CTVD de Polesgo. Le modèle fait l'hypothèse qu'il n'y a aucune production de biogaz lors de la $1^{\text {ère }}$ année (2005) d'enfouissement des déchets. La fermentation anaérobie n'avait donc pas encore démarré. En conséquence, la production de biogaz débute en 2006 et la contribution du CTVD aux émissions des GES s'élevait à 7801 téq- $\mathrm{CO}_{2}$. Année après année, avec l'apport de nouveaux déchets, l'empreinte carbone augmente. En 2019, l'empreinte 
carbone du CTVD est de 125539 téq- $\mathrm{CO}_{2}$ et elle atteint 901267 téqCO ${ }_{2}$ depuis l'ouverture du CTVD de Polesgo en 2005. Cette importante contribution à l'effet de serre s'explique d'une part par la forte teneur en matière organique des déchets enfouis au CTVD de Polesgo (Haro et al., 2018) et les conditions météorologiques notamment la température d'autre part. Beaucoup d'étude ont rapporté que la température a une influence considérable dans le processus de biodégradation des déchets (Allam, 2015 ; Bastone et al., 2002 ; Rouez, 2008). Selon Batstone et al., 2002, l'augmentation de la température (de 10 à $60^{\circ} \mathrm{C}$ ) augmente les vitesses réactionnelles de la degradation de la fraction organique des déchets comme l'a prédit la loi d'Arrhenius. Ces paramètres sont favorables à une bonne production d'un biogaz riche en $\mathrm{CH}_{4}$.

Il ressort de la présente étude que la contribution maximale du CTVD aux émissions des GES serait atteinte en 2026 (196534 téq- $\mathrm{CO}_{2}$ ), un an après la fermeture prévue du CTVD. A cette date, la contribution totale s'élèverait à plus de 2 millions de téq- $\mathrm{CO}_{2}$. Une empreinte carbone plus importante un an après la fermeture du CTVD est en accord avec les études antérieures selon le modèle LandGEM (Chalvatzaki et Lazaridis, 2010). Après 2026 la contribution va décroître de manière exponentielle et tendre vers 0 après 2145, même si plus de 100 ans après sa fermeture, le CTVD contribuera toujours aux émissions des GES.

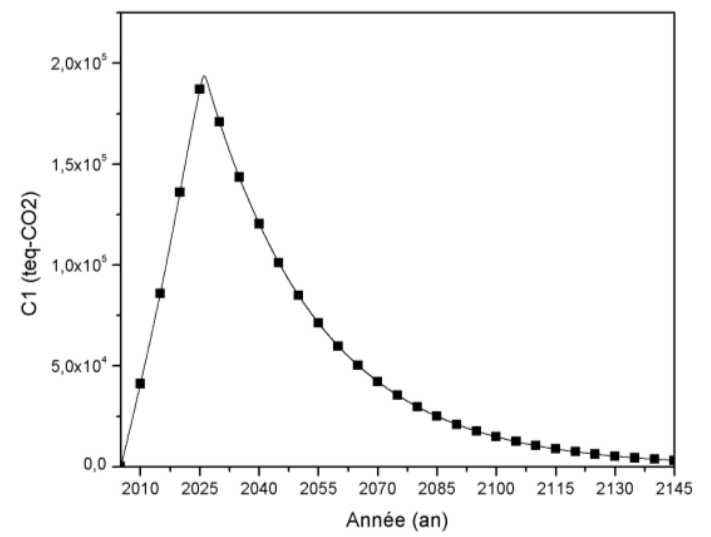

Fig. 2. Evolution de l'empreinte carbone CTVD de Polesgo

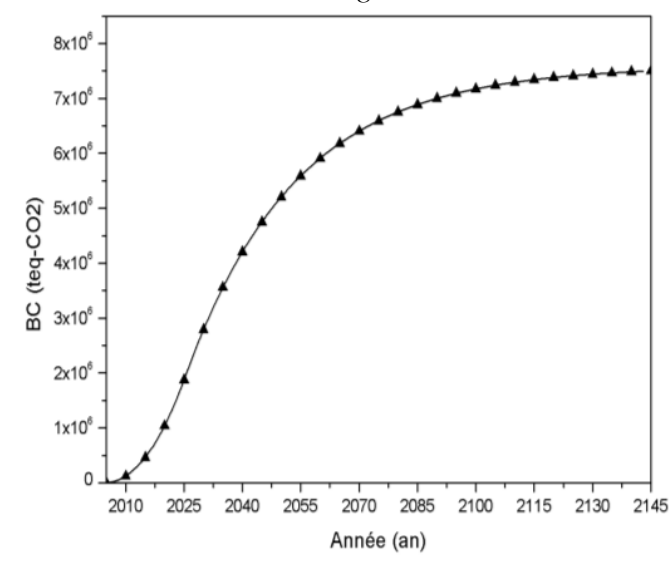

Fig. 3. Contribution cumulée des émissions des GES du CTVD de Polesgo

\section{CONCLUSION ET PERSPECTIVES}

L'enfouissement des déchets solides ménagers au CTVD de Polesgo a occasionné une émission d'environ 125539 téq- $\mathrm{CO}_{2}$ en 2019 et plus 901267 téq$\mathrm{CO}_{2}$ depuis son ouverture en 2005, selon le modèle LandGEM. Ces énormes quantités de GES sont liés au fait que les déchets enfouis au CTVD sont fortement organiques et qu'aucun dispositif de valorisation ou de torchère n'est disponibles à ce jour au CTVD dont la conception intégrait pourtant un volet valorisation. Il sera plus judicieux de prospecter d'autres options de traitement telles que la valorisation organique et matière au détriment de l'enfouissement, dans le contexte de lutte contre les changements climatiques. A défaut de ces autres options, un simple brulage du biogaz capté permettra de transformer le $\mathrm{CH}_{4}$ $(\mathrm{PRG}=28)$ en $\mathrm{CO}_{2}(\mathrm{PRG}=1)$. Ce qui contribuera à la réduction de l'empreinte carbone du CTVD de Polesgo. Ces types d'exercices sont à encourager dans d'autres centres de traitement des déchets dans les villes des pays en développement afin de permettre une amélioration de la gestion des déchets.

Pour aller plus loin dans cette réflexion il sera plus intéressant de comparer plusieurs scénarii de gestion des déchets afin de fournir un scénario bas carbone aux autorités en charge de la gestion des déchets solides municipaux.

\section{REFERENCES}

Alexander, A., Burklin, C. and Singleton, A., 2005. Landfill Gas Emissions Model (LandGEM) Version 3.02 User's Guide, USA.

Allam, N., 2015. Estimation des émissions surfaciques du biogaz dans une installation de stockage des déchets non dangereux. Thèse de doctorat; Ecoles des Mines de Nantes, France. Disponible à : https://tel.archives-ouvertes.fr/tel-01148512.

Batstone D.J., Keller J., Angelidaki I., Kalyuzhnyi S. V., Pavlostathis S. G., Rozzi A., Sanders WTM, Siegriest H., Vavilin V. A., Anaerobic Digestion Model $\quad \mathrm{N}^{\circ} 1 \quad$ (ADM1). International Water Association Scientific and Technical Report $\mathrm{n}^{\circ} 13$, London, UK, IWA Publishing, 2002, 68p.

Bogner, J., Pipatti, R., Hashimoto, S., Diaz, C., Mareckova, K., Diaz, L., Kjeldsen, P., Monni, S., Faaij, A., Gao, Q., Zhang, T., Ahmed, A. M., Sutamihardja, R. T. M. and Gregory R., 2008. Mitigation of global greenhouse gas emissions from waste: conclusions and strategies from the Intergovernmental Panel on Climate Change (IPCC) FourthAssessment Report. Working Group III (Mitigation). Waste Management \& Research, 26 (11), pp.11-32. http://doi.org/10.1177/0734242x 07088433 
Chalvatzaki E. et Lazaridis M., 2010.Estimation of greenhouse gas emissions from landfills: application to the Akrotiri landfill site (Chania, Greece). Global NEST Journal, 12, pp.108-116.

Guerrero, L.-A., Mass, G., Hogland, W., 2013. Solid wastes management challenges for cities in developing countries. Waste Management, 33, 220232

Gunnar, B., Asa, D. and Bo, H. S., 2000. Methane Fluxes from a Swedish Landfill Determined by Geostatistical Treatment of Static Chamber Measurements., 34(18), pp.4044-4050

Haro, K., Ouarma, I., Nana, B., Bere, A., and Koulidiati, J., 2017. In-situ measurement and theoretical calculations of annual biogas generation: case of Polesgo landfill at Burkina Faso, Proceedings Sardinia 2017 / Sixteenth International Waste Management and Landfill Symposium/ 2 - 6 October 2017, S. Margherita di Pula, Cagliari, Italy/ (c) 2017 by CISA Publisher, Italy.

Haro, K., Ouarma, I., Nana, B., Bere, A., and Koulidiati, J., 2018. Characterization and Potential Recovery of Household Solid Waste in the City of Ouagadougou (Burkina Faso), Journal of Environmental Protection, 09(04):309-324, Available at: http://dx.doi.org/10.4236/jep.2018.94021.

INSD (Institut National de la Statistique et de la démographie), 2017. Projection démographique des communes du Burkina Faso de 2007 à 2020, Ouagadougou / Burkina Faso. Available at: http://www.insd.bf/n/contenu/autres_publications / Projection_com_Burkina_2007_2020.pdf
IPCC, 2013. Climate Change 2013: The Physical Science Basis, Contribution of Working Group I to the Fifth Assessment Report of the Intergovernmental Panel on Climate Change, 1535.

Krautwurst, S., Gerilowski, K., Jonsson, H. H., Thompson, D. R., Kolyer, R. W., Iraci, L. T., Thorpe, A. K., Horstiann, M., Eastwood, M. and Leifa, I., 2017. Methane emissions from a Californian landfill, determined from airborne remote sensing and in situ measurements. Atmospheric Measurement Techniques, 10, pp.3429-3452.

Pham, C. H., Saggar, S., Vu, C. C., Tate, K. R, Tran, T. T., Luu, T. T., Ha, H. T., Nguyen H. L. T. and Sommer, S. G., 2017. Biogas production from steermanures in Vietnam: Effects of feed supplements and tannin contents. Waste Management, 69, pp.492-497. Available at : https://doi.org/10.1016/ j.wasman.2017. 08.002.

Rouez, M., 2008. Dégradation anaérobie de déchets solides: caractérsation, facteurs d'influence et modélisation. Thèse de doctorat ; Institut National des Sciences Appliquées de Lyon, France. Disponible à http://docinsa2.insalyon.fr/these/2008/rouez/these.pdf

Tezanou, J., Koulidiati, J., Sougoti, M., Barhe, J. A., Kafando, P., Segda, B., G et Joulain, P., 2003. Caractérisation expérimentale des ordures ménagères des pays en voie de développement (PED): Cas de la ville de Ouagadougou (Burkina Faso). Annales de l'Université de Ouagadougou Série C: Sciences de la Vie et de la Matière, 001, pp.54-83.

UNEP (Programme des Nations Unies pour l'Environnement), 2010. Waste and Climate Change, Kenya. Available at: http://www.unep.or.jp. 